\title{
A New Smoothing Nonmonotone Trust Region Method for Solving Nonlinear Complementarity Problems
}

\author{
Ji Ying \\ School of Management \\ Harbin Institute of Technology \\ Academy of Fundamental and Interdisciplinary Science \\ Harbin Institute of Technology \\ Harbin, China
}

\author{
li yijun \\ School of Management \\ Harbin Institute of Technology \\ Harbin, China
}

\begin{abstract}
In this paper, we propose one new smoothing nonmonotone trust region method for solving nonlinear complementarity problems with $P_{0}-$ functions. First, the nonlinear complementarity problem $(\mathrm{NCP})$ is reformulated as a nonsmooth equation. Then on the basis of the reformulation, a smoothing nonmonotone trust region algorithm via a line

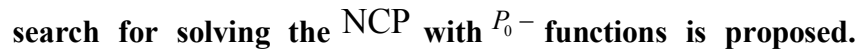
When a trial step is not accepted, the method does not resolve the trust region subproblem but generates a iterative point whose steplength is generated by a formula. We prove that every accumulation point of the sequence generated by the algorithm is a solution of the $\mathrm{NCP}$. Under a nonsingularity condition, the superlinear convergence of the algorithm is established without the strict complementarity condition.
\end{abstract}

Keywords-NCP, Trust region method, Fixed steplength, Nonmonotone technique

\section{INTRODUCTION}

The nonlinear complementarity problem, denoted by $\operatorname{NCP}(F)$, is to find a vector $x \in R^{n}$ such that

$$
x \geq 0, F(x) \geq 0, x^{T} F(x)=0
$$

where $F: R^{n} \rightarrow R^{n}$ is assumed to be continuously differentiable.

In this paper, we propose and analyze a new smoothing nonmonotone trust region algorithm that combines elements of trust region methods with elements of line search methods. The new algorithm retains the quick convergence and stability of trust region methods, while significantly decreasing the average cost per iteration of the method. We first reformulate the $\mathrm{NCP}$ as a system of semismooth by using the Fischer-Burmeister function, then using Kanzow's [1] smooth approximation function to construct the smooth operator, we propose a smoothing nonmonotone trust region algorithm via a line search for solving the NCP with $P_{0}$ - functions. When a trial step is not accepted, the method does not resolve the trust region subproblem but generates a iterative point whose steplength is generated by a formula. We prove that every accumulation point of the sequence generated by the algorithm is a solution of the NCP.
Compared with the method in [2], the superlinear convergence of the proposed algorithm is established without the strict complementarity condition.

We will use Kanzow's [1] smooth approximation

$$
\varphi_{\varepsilon}(a, b):=\sqrt{a^{2}+b^{2}+2 \varepsilon}-a-b, \varepsilon>0
$$

for the Fischer-Burmeister function. The corresponding smooth operator $\Phi_{\varepsilon}: R^{n} \rightarrow R^{n}$ is defined by

$$
\begin{gathered}
\Phi_{\varepsilon}(x)=\left(\begin{array}{l}
\varphi_{\varepsilon}\left(x_{1}, F_{1}(x)\right) \\
\ldots \\
\varphi_{\varepsilon}\left(x_{n}, F_{n}(x)\right)
\end{array}\right) . \\
\Psi_{\varepsilon}(x):=\frac{1}{2} \Phi_{\varepsilon}(x)^{T} \Phi_{\varepsilon}(x)
\end{gathered}
$$

Denote can be approximated by the following nonlinear least square problems,

$$
\min _{x \in R^{n}} \Psi_{\varepsilon}(x)
$$

By the above discussions, we have that when $\varepsilon \rightarrow 0$ the problem (3) is equivalent to $\mathrm{NCP}(F)$.

\section{The Algorithm}

Define

$$
\begin{aligned}
& Q_{k}(s):=\frac{1}{2}\left\|\Phi_{\varepsilon_{k}}\left(x_{k}\right)+\nabla \Phi_{\varepsilon_{k}}\left(x_{k}\right)^{T} s\right\|^{2} \\
& =\Psi_{\varepsilon_{k}}\left(x_{k}\right)+\nabla \Psi_{\varepsilon_{k}}\left(x_{k}\right)^{T} s+\frac{1}{2} s^{T} \nabla \Phi_{\varepsilon_{k}}\left(x_{k}\right) \nabla \Phi_{\varepsilon_{k}}\left(x_{k}\right)^{T} s
\end{aligned}
$$

Then the trial step $S_{k}$ will be obtained by solving the following trust region subproblem,

$$
\min \left\{Q_{k}(s) \mid\|s\| \leq \Delta_{k}\right\}
$$

where $\Delta_{k}$ is called trust region radius. Let $S_{k}$ be the solution of the subproblem (6).

Then either $x_{k}+s_{k}$ is accepted as a new iteration point or the trust-region radius is reduced according to a 
comparison between the actual reduction of the objective function

$$
\operatorname{ared}_{k}\left(s_{k}\right):=\Psi_{\varepsilon_{l(k)}}\left(x_{l(k)}\right)-\Psi_{\varepsilon_{k}}\left(x_{k}+s_{k}\right)
$$

and the reduction predicted by the model

$$
\operatorname{pred}_{k}\left(s_{k}\right):=Q_{k}(0)-Q_{k}\left(s_{k}\right)
$$

i.e.,

$r_{k}:=\frac{\operatorname{ared}_{k}\left(s_{k}\right)}{\operatorname{pred}_{k}\left(s_{k}\right)}$

where

$$
\Psi_{\varepsilon_{l(k)}}\left(x_{l(k)}\right)=\max _{0 \leq j \leq m(k)}\left\{\Psi_{\varepsilon_{k-j}}\left(x_{k-j}\right)\right\}, k=0,1,2, \ldots,
$$

and

$$
m(k):= \begin{cases}0, & \text { if } r_{k} \geq c_{2} \\ \min \{m(k-1)+1, M\}, & \text { otherwise, }\end{cases}
$$

$m(0):=0, M \geq 1$ is an integer constant. That is, if the reduction in the objective function is satisfactory, then we finish the current iteration by taking

$x_{k+1}:=x_{k}+s_{k}$ and adjusting the trust-region radius; otherwise a new iterative point by $x_{k+1}=x_{k}+\alpha_{k} s_{k}$ is generated, where $\alpha_{k}$ is a steplength generated by

$$
\alpha_{k}=-\frac{\delta \Psi_{\varepsilon_{k}}\left(x_{k}\right)^{T} s_{k}}{s_{k}{ }^{T} \Phi_{\varepsilon_{k}}\left(x_{k}\right) \Phi_{\varepsilon_{k}}\left(x_{k}\right)^{T} s_{k}}
$$

here $\delta>0$ is a constants.

Algorithm 1(The smoothing nonmonotone trust region algorithm via a line search for $\mathrm{NCP}(F)$,

Step $\quad 0$. Choose parameters $0<c_{2}<c_{1}<1$, $0<c_{3}<1<c_{4}, \Delta_{\text {min }}>0, v>0,0<\eta<1,0<\mu<1$. Give a starting point $x_{0} \in R^{n}$, an integer constant $M \geq 0$ and an initial trust-region radius $\Delta_{\text {min }} \leq \Delta_{0}$; set $\Gamma_{0}:=(1+\mu)\left\|\Phi\left(x_{0}\right)\right\|, \quad \beta_{0}:=\left\|\Phi\left(x_{0}\right)\right\|, \quad \kappa:=\sqrt{2 n}$, $\varepsilon_{0}:=\left(\left(\mu /\left(2 \Gamma_{0} \kappa\right)\right) \beta_{0}^{2}\right)^{2} \quad, \quad m(0):=0, M \geq 1 \quad$ and $k:=0$.

Step 1. Compute the solution $s_{k} \in R^{n}$ of the subproblem (6).

Step 2. Compute $\operatorname{ared}_{k}\left(s_{k}\right), \operatorname{pred}_{k}\left(s_{k}\right)$ and $r_{k}:=\frac{\operatorname{ared}_{k}\left(s_{k}\right)}{\operatorname{pred}_{k}\left(s_{k}\right)}$

If $k \geq 1$, compute $m(k)$ by (10). If $r_{k} \geq c_{2}$, then

$$
x_{k+1}:=x_{k}+s_{k}
$$

$$
\Delta_{k+1}:=\left\{\begin{array}{l}
\max \left[c_{4} \Delta_{k}, \Delta_{\min }\right], \quad \text { if } r_{k}>c_{1} \\
\max \left[\Delta_{k}, \Delta_{\min }\right], \quad \text { otherwise }
\end{array}\right.
$$

Otherwise generate ${ }^{\alpha_{k}}$ by (11) and set

$$
x_{k+1}=x_{k}+\alpha_{k} s_{k}, \quad \Delta_{k}:=c_{3}\left\|s_{k}\right\|
$$

$$
\text { Step 3. If }\left\|\nabla \Psi\left(x_{k+1}\right)\right\|=0 \text {, stop. }
$$$$
\text { Step 4. If }
$$

$$
\left\|\Phi\left(x_{k+1}\right)\right\| \leq \max \left\{\eta \beta_{k}, \mu^{-1}\left\|\Phi\left(x_{k+1}\right)-\Phi_{\varepsilon_{k}}\left(x_{k+1}\right)\right\|\right\},
$$$$
\text { then set } \beta_{k+1}:=\left\|\Phi\left(x_{k+1}\right)\right\| \text { and choose }{ }^{\varepsilon_{k+1}} \text { such that }
$$$$
0<\varepsilon_{k+1} \leq \min \left\{\left(\frac{\mu}{2 \Gamma_{0} \kappa} \beta_{k+1}^{2}\right), \frac{\varepsilon_{k}}{4}, \bar{\varepsilon}\left(x_{k+1}, \nu \beta_{k+1}\right)\right\}
$$

where $^{\bar{\varepsilon}(\cdot, \cdot)}$ is defined in Lemma 2.3 [3]; otherwise, let $\beta_{k+1}:=\beta_{k}$ and $\varepsilon_{k+1}:=\varepsilon_{k}$.

Step 5. Set $k:=k+1$, and go to Step 1 .

Lemma 1 Suppose that $F$ is a $P_{0}$ function, and Algorithm 2.1 does not stop finitely, then $s_{k} \neq 0$ and furthermore $\operatorname{pred}_{k}\left(s_{k}\right)>0, \forall k$.

Theorem 2 If $F$ is a $P_{0}$ function, then the sequence $x_{k}$ generated by Algorithm 2.1 remains in the level set $L_{0}:=\left\{x \in R^{n} \mid \Psi(x) \leq \Psi\left(x_{0}\right)\right\}$ with $\delta_{0}=\left(\frac{1+\mu}{1-\mu}\right)^{2}$

Theorem 3 Suppose that $F$ is a $P_{0}$ function. Then Algorithm 2.1 is well defined.

\section{Global CONVERGENCE}

Theorem 1 Suppose that $F_{\text {is a }} P_{0}$ function. Then there exists a positive constant $\delta_{2}$ such that

$$
\operatorname{pred}_{k}\left(s_{k}\right) \geq \delta_{2}\left\|\nabla \Psi_{\varepsilon_{k}}\left(x_{k}\right)\right\| \min \left[\Delta_{k}, \frac{\left\|\nabla \Psi_{\varepsilon_{k}}\left(x_{k}\right)\right\|}{\left\|\nabla \Phi_{\varepsilon_{k}}\left(x_{k}\right)\right\|^{2}}\right]
$$

for all $k$, where $S_{k}$ is the solution to (2.3).

Lemma 2 Suppose that $F_{\text {is a }} P_{0}$ function. Then there exists a positive constant $\delta_{3}$ such that

$\left|\Psi_{\varepsilon_{k}}\left(x_{k}\right)-\Psi_{\varepsilon_{k}}\left(x_{k}+s_{k}\right)-\operatorname{pred}_{k}\left(s_{k}\right)\right| \leq \delta_{3}\left\|s_{k}\right\|^{2}, \forall k$

Theorem 3 Suppose that $F$ is a $P_{0}$ function, and there exists $\varepsilon>0$ such that $\left\|\nabla \Psi_{\varepsilon_{k}}\left(x_{k}\right)\right\| \geq \varepsilon$ for all $k$. Then there exists a constant $\delta_{4}>0$ such that 


$$
\Psi_{\varepsilon_{l(k)}}\left(x_{l(k)}\right)-\Psi_{\varepsilon_{k+1}}\left(x_{k+1}\right) \geq \delta_{4} \min \left\{\Delta_{k}, \frac{\varepsilon}{\left\|\nabla \Phi_{\varepsilon_{k}}\left(x_{k}\right)\right\|^{2}}\right\}
$$

holds for all $k$.

Lemma 4 Suppose that $F$ is a $P_{0}$ function, and there exists $\varepsilon>0$ such that $\left\|\nabla \Psi_{\varepsilon_{k}}\left(x_{k}\right)\right\| \geq \varepsilon$ for all $k$. Then there exists $v \in(0,1)$ such that

$$
\lim _{k \rightarrow \infty}\left\{v \Delta_{k}, \frac{\varepsilon}{Z_{k}}\right\}=0 \text {, where } Z_{k}:=1+\max _{1 \leq i \leq k}\left\|\nabla \Phi_{\varepsilon_{i}}\left(x_{i}\right)\right\|^{2} .
$$

Lemma 5 Suppose that $F$ is a $P_{0}$ function, and there exists $\varepsilon>0$ such that $\left\|\nabla \Psi_{\varepsilon_{k}}\left(x_{k}\right)\right\| \geq \varepsilon$ for all $k$. Then the following inequality

$\left\|s_{k}\right\| \geq \min \left\{1, \delta_{2} \varepsilon\left(1-c_{2}\right)\right\} / Z_{k} \quad, \quad$ holds for $k \in J$ sufficiently large.

Lemma 6 Suppose that $F$ is a $P_{0}$ function, and there exists $\varepsilon>0$ such that $\left\|\nabla \Psi_{\varepsilon_{k}}\left(x_{k}\right)\right\| \geq \varepsilon$ for all $k$. Then the following inequality

$$
\Delta_{k} \geq c_{3} \min \left\{1, \delta_{2} \varepsilon\left(1-c_{2}\right)\right\} / Z_{k} \text {, holds for sufficiently }
$$
large $k$.

Theorem 7 Assume that $F$ is a $P_{0}$ function. Let $\left\{x_{k}\right\}$ be a sequence generated by Algorithm 2.1. Then every accumulation point of the sequence $\left\{x_{k}\right\}$ is a solution of $\operatorname{NCP}(F)$

\section{LOCAL CONVERGENCE}

Theorem 1 Suppose $F$ is a $P_{0}$-function. If for an accumulation point $x^{*}$ of the sequence $\left\{x_{k}\right\}_{k \in \hat{K}}$, all $V=\partial_{C} \Phi\left(x^{*}\right)$ are nonsingular, then the following statements hold:

1) The whole sequence $\left\{x_{k}\right\}$ converges to $x^{*}$.

2) Eventually all iterations are successful and the trust region radius in subproblem (3.3) is inactive; i.e., for all $\mathrm{k}$ sufficiently large, we have

$$
x_{k+1}=x_{k}+s_{k}, s_{k}=-\left(\Phi_{\varepsilon_{k}}^{\prime}\left(x_{k}\right)\right)^{-1} \Phi_{\varepsilon_{k}}\left(x_{k}\right)
$$

3) The sequence $\left\{x_{k}\right\}$ converges to $x^{*}$ superlinearly.

\section{ACKNOWLEDGEMENT}

This work was supported by both China Post-doctoral Science Foundation (No.01107172) and Heilongjiang Province Post-doctoral Science Foundation (No. 01106961). Email address : jiying_1981@126.com

\section{REFERENCES}

[1] C. Kanzow, Some Noninterior Continuation Methods for Linear Complementarity Problems[J], SIAM J. Matrix Anal. Appl., 1996, PP 851-868.

[2] J.W. Zhang and D.H. Li, A norm descent BFGS method for solving KKT systems of symmetric variational inequality problems, Optimization Methods and Software, 2007, PP 237-252.

[3] S.J. Qu, M. Goh, X.J. Zhang, A new hybrid method for nonlinear complementarity problems, Comput Optim Appl, 2011, DOI 10.1007/s10589-009-9309-7. 